\title{
Transcriptome sequencing and comparative analysis of adult ovary and testis identify potential gonadal maintenance- related genes in Mauremys reevesii with temperature- dependent sex determination
}

Lei Xiong ${ }^{1,2}$, Jinxiu Dong ${ }^{1}$, Hui Jiang ${ }^{1}$, Jiawei Zan ${ }^{1}$, Jiucui Tong ${ }^{1,2}$, Jianjun Liu ${ }^{1}$, Meng Wang ${ }^{1}$, Liuwang Nie ${ }^{\text {Corresp. } 1}$

${ }^{1}$ Life Science College of Anhui Normal University, Provincial Key Lab of the Conservation and Exploitation Research of Biological Resources in Anhui, Wuhu, Anhui, P.R. China

2 Biochemistry Department of Wannan Medical College, Provincial Key Laboratory of Biological Macro-molecules Research, Wuhu, Anhui, P.R. China

Mauremys reevesii is a classical organism with temperature-dependent sex determination (TSD). Gonad development in early life has recently received considerable attention but gonadal maintenance after sex differentiation in turtles with TSD remains a mystery. In this study, we sequenced the transcriptomes for the adult testis and ovary using RNA-seq, and 36,221 transcripts were identified. In total, 1,594 differentially expressed genes (DEGs) were identified where 756 DEGs were upregulated in the testis and 838 DEGs were upregulated in the ovary. Gene Ontology and Kyoto Encyclopedia of Genes and Genomes pathway analysis suggested that the TGF-beta signaling pathway and Hedgehog signaling pathway have important roles in testis maintenance and spermatogenesis, whereas the Hippo signaling pathway and Wnt signaling pathway are likely to participate in ovary maintenance. We determined the existence of antagonistic networks containing significant specific-expressed genes (SEGs) and pathways related to gonadal maintenance and gametogenesis in the adult gonads of $M$. reevesii. The candidate gene Fibronectin type 3 and ankyrin repeat domains 1 (FANK1) might be involved with the regulation of testis spermatogenesis. 


\section{Transcriptome sequencing and comparative analysis of}

3 adult ovary and testis identify potential gonadal

4 maintenance-related genes in Mauremys reevesii with

5 temperature-dependent sex determination

6 Lei Xiong ${ }^{1,2}$, Jinxiu Dong ${ }^{1}$, Hui Jiang ${ }^{1}$, Jiawei Zan ${ }^{1}$, Jiucui Tong ${ }^{1,2}$, Jianjun Liu ${ }^{1}$, Meng Wang ${ }^{1}$,

7 Liuwang $\mathrm{Nie}^{1}$

8

$9 \quad{ }^{1}$ Life Science College of Anhui Normal University, Provincial Key Lab of the Conservation and

10 Exploitation Research of Biological Resources in Anhui, Wuhu, Anhui, P.R. China

$11{ }^{2}$ Biochemistry Department of Wannan Medical College, Provincial Key Laboratory of

12 Biological Macro-molecules Research, Wuhu, Anhui, P.R. China

13

14 Corresponding Author:

15 Liuwang $\mathrm{Nie}^{1}$

Email address: 1wnie@mail.ahnu.edu.cn

17

18

19

\section{Abstract}

Mauremys reevesii is a classical organism with temperature-dependent sex determination (TSD).

Gonad development in early life has recently received considerable attention but gonadal maintenance after sex differentiation in turtles with TSD remains a mystery. In this study, we sequenced the transcriptomes for the adult testis and ovary using RNA-seq, and 36,221 transcripts were identified. In total, 1,594 differentially expressed genes (DEGs) were identified where 756 DEGs were upregulated in the testis and 838 DEGs were upregulated in the ovary. Gene Ontology and Kyoto Encyclopedia of Genes and Genomes pathway analysis suggested that the TGF-beta signaling pathway and Hedgehog signaling pathway have important roles in testis 
27 maintenance and spermatogenesis, whereas the Hippo signaling pathway and Wnt signaling

28 pathway are likely to participate in ovary maintenance. We determined the existence of

29 antagonistic networks containing significant specific-expressed genes (SEGs) and pathways

30 related to gonadal maintenance and gametogenesis in the adult gonads of $M$. reevesii. The

31 candidate gene Fibronectin type 3 and ankyrin repeat domains 1 (FANK1) might be involved

32 with the regulation of testis spermatogenesis.

\section{Introduction}

Mauremys reevesii, also called Reeves' pond turtle, is one of the most common and

37 widespread semiaquatic turtles in East Asia (Yin et al., 2016). This species is a classical 38 organism with temperature-dependent sex determination (TSD). Previous studies developed a gene expression model for the embryonic gonads in reptiles with TSD (Yatsu et al., 2016;

40 Czerwinski et al., 2016), and many of the sex-related genes have also been identified, e.g., DMRT1 in Pelodiscus sinensis and Trachemys scripta (Sun et al., 2017; Ge et al., 2017), and CYP19A1 in T. scripta (Matsumoto et al., 2013). In species with significant sex chromosomal differentiation, gonadal maintenance after sex differentiation depends on the expression of $\mathrm{XX} / \mathrm{XY}$ or ZZ/ZW genes. However, species with TSD have almost identical genetic materials in the males and females. Thus, the mechanisms responsible for functional maintenance and gametogenesis during the sexual maturity period remain a mystery. Transcriptome sequencing provides a general representation of most of the transcripts that are expressed in specific cells or organs under particular conditions and stages (Liu et al., 2018), and it is considered the best option for identifying candidate genes in organisms that lack a reference genome (Yin et al., 2016). 
In this study, we performed a comparative transcriptome analysis of the adult gonads (testis and ovary) in $M$. reevesii in order to identify molecular signaling cascades and gene expression networks. We also annotated all of the sequenced transcripts and enriched differentially expressed genes (DEGs), and pathways related to gonadal maintenance in these two sexual organs. These results provide crucial genomic information to facilitate further research into the regulatory mechanisms related to gonadal maintenance and gametogenesis during sexual maturity in turtle species with TSD.

\section{Materials \& Methods}

Ethical approval

61 Procedures involving animals and their care were approved by the Animal Care and Use

62 Committee of Anhui Normal University (\#20170612).

\section{Sample collection and RNA extraction}

64 Six adult turtles $(3 \hat{o}, 3 \hat{f})$ were cultivated under the same rearing conditions (i.e., maintenance in a room at temperatures of $10-30^{\circ} \mathrm{C}$ ) in the Provincial Key Laboratory for the Conservation and Exploitation Research of Biological Resources in Wuhu $\left(31^{\circ} 33^{\prime} \mathrm{N}, 118^{\circ} 370^{\prime} \mathrm{E}\right.$, southeast of China) in 2017. The turtles were aged more than six years old in the sexual maturity stage, and the sexes of the samples were determined by dissection. Gonad samples (testicles and ovaries) were collected separately and dissected (Figure 1). The gonad samples were flash frozen in liquid nitrogen and stored at $-80^{\circ} \mathrm{C}$ until RNA was extracted (Yin et al., 2016).

RNA was extracted from each sample with TRIzol reagent (Invitrogen, Carlsbad, CA, USA) according to the manufacturer's instructions. To effectively remove the genomic DNA, we added RNase-free DNase I (Takara, Dalian, China) to the reaction mixture for at least $10 \mathrm{~min}$. The extracted RNA was quantified with the Nanodrop system (Thermo, Wilmington, DE, USA) and 
75 the fragment size distribution were checked by $1.5 \%$ agarose gel electrophoresis. RNA from

76 each sample was used for cDNA synthesis and sequencing.

77 cDNA library construction and sequencing

78 We used $15 \mu$ g of total RNA for cDNA library construction using a TruSeq $\mathbb{R}$ RNA LT Sample

79 Prep Kit v2 (Illumina, San Diego, CA, USA) according to the manufacturer's protocol. Six

80 cDNA libraries $(3 \hat{\delta}, 3$ 우 $)$ were constructed by performing end-repair, $3^{\prime}$-end adenylation, and

81 adapter ligation and enrichment. Sequencing was performed using the Illumina Hiseq 2500

82 platform by Genergy Bio-technology Co. Ltd (Shanghai, China). All sequence reads have been

83 deposited with the NCBI (GenBank accession No. SRP153785).

84 Sequence data processing and de novo assembly

85 The raw reads generated by Illumina sequencing were subjected to a screening process where the 86 adapter sequences and low-quality reads were trimmed from the raw reads using Trim Galore 87 v0.5.0 (http://www.bioinformatics.babraham.ac.uk/projects/trim_galore/) software. FastQC 88 v0.11.8 (http://www.bioinformatics.babraham.ac.uk/projects/fastqc/) was used to check the 89 quality of the pretreated data. Trinity v2.8.4 (https://github.com/trinityrnaseq/trinityrnaseq/

90 releases/tag/Trinity-v2.8.4) was used to perform de novo assembly with the default parameters

91 (Grabherr et al., 2011). The clean high quality data were spliced to obtain the reference 92 sequences (transcripts) for subsequent analyses.

93 Gene annotation

94 Multiple public databases were used for homology annotation, including non-redundant 95 nucleotide and protein sequences (NT/NR) (http://www.ncbi.nlm.nih.gov), Swiss-Prot 96 (http://www.expasy.ch/sprot), euKaryotic Orthologous Groups (KOG) (ftp://ftp.ncbi.nih.gov/ $97 \mathrm{pub} / \mathrm{COG} / \mathrm{KOG} /$ ) based on BLASTX (NCBI blast 2.2.28) with an e-value of 1e-5 (Altschul et al., 
98 1990), Gene Ontology (http://www.geneontology.org/) based on Blast2GO v2.5 with an e-value

99 of 1e-6, and Kyoto Encyclopedia of Genes and Genomes (KEGG; http://www.genome.jp/kegg)

100 based on KAAS with an e-value of 1e-10 (Kanehisa et al., 2008). InterProScan v5.31-70.0

101 (http://www.ebi.ac.uk/interpro/download.html) was also used to predict the functional domains,

102 signal peptides, and other protein characteristics by BLASTing against the Conserved Domain

103 Database Interpro under the default parameters (Zdobnov \& Apweiler, 2001). Gene Ontology

104 (GO) terms were assigned to three categories (biological process, molecular function, and

105 cellular component) (Conesa et al., 2005). The KEGG Automatic Annotation Server was

106 employed for KEGG annotation (Moriya et al., 2007).

107 DEG analysis

108 Clean reads were aligned using Bowtie 2 v2.3.4.3 (http://bowtie-bio.sourceforge.net/

109 bowtie2/index.shtml) (Langmead, 2010). Transcript abundance was estimated using RSEM

110 v1.3.1 (http://deweylab.github.io/RSEM/) in the Trinity package ( $L i \&$ Dewey, 2011). The

111 expression profiles were detected for the transcripts using the gene expression method as the

112 fragments per kilobase of transcript per million mapped reads (Trapnell et al., 2010). For

113 samples with three biological replicates, the differential expression of unigenes between males

114 and females was analyzed using the R (v3.4.2) package DESeq (Anders \& Huber, 2010). Based

115 on the negative binomial distribution model, DESeq provides statistical routines for determining

116 differential expression in digital gene expression data ( $H u$ et al., 2018). The statistical $P$-values

117 were adjusted using Benjamini and Hochberg's approach for controlling the false discovery rate

118 (Benjamini \& Hochberg, 1995). Unigenes with a corrected $P$-value $<0.05$ and $\log _{2}$ FoldChange $\geq$

1191 or $\log _{2}$ FoldChange $\leq-1$ were considered to be DEGs. Significant specific-expressed genes 
120 (SEGs) were identified from DEGs. Visualizations of the analyses, including the heatmap,

121 volcano, GO, and KEGG enrichment results, were performed in the R package with ggplot2.

122 Validation of transcriptome data by real-time qRT-PCR

123 To verify the accuracy of the transcriptome data, three DEGs and nine SEGs were selected

124 randomly for real-time qRT-PCR. All of the reactions were performed using three technical

125 replicates and three biological replicates to validate the reliability of the results. A Real-time

126 Detection System (Bio-Rad C1000 Thermal Cycler, Hercules, CA, USA) was employed to

127 perform real-time qRT-PCR. The PCR reaction system comprised the following: $10 \mu \mathrm{L}$

128 SuperReal PreMix Plus (Tiangen, Beijing, China), $0.5 \mu \mathrm{L}$ of each primer (10 $\mu \mathrm{M}$ in total), $1 \mu \mathrm{L}$

129 of template cDNA, and $8 \mu \mathrm{L}$ of RNase-free $d_{d H_{2}} \mathrm{O}$. The expression profile was detected in

130 triplicate wells under the following protocol: $95^{\circ} \mathrm{C}$ for $15 \mathrm{~min}, 40$ cycles for $10 \mathrm{~s}$ at $95^{\circ} \mathrm{C}$ and 20

$131 \mathrm{~s}$ at $58^{\circ} \mathrm{C}$, and $30 \mathrm{~s}$ at $72^{\circ} \mathrm{C}$, before finally ramping from $65-95^{\circ} \mathrm{C}$ at $0.5^{\circ} \mathrm{C}$ per $5 \mathrm{~s}$ to generate a

132 melting curve. The turtle GADPH gene was selected as the internal control gene. The relative

133 expression level of each contig in different samples was calculated using the $2^{-\Delta \Delta C T}$ method

134 (Livak \& Schmittgen, 2001). The primers employed are listed in the supplemental materials 135 (Table S1).

136

137 Results

138 Sequencing and de novo assembly

139 Six libraries $(3 \stackrel{0}{3}, 3$ ) $)$ were established and sequenced (Table 1). In total, 508,785,280 paired-

140 end raw reads with lengths over 100 bp were generated. We obtained 496,163,850 (97.57\%)

141 high-quality reads with an average length of $145.87 \mathrm{bp}$. The de novo assembly results yielded

142557,690 isoforms, which clustered into 437,767 unigenes with an N50 of 631 bp. The GC

143 content of the entire final assembly was $53.46 \%$. 


\section{Unigene functional annotation}

145 Among the total of 437,767 unigenes, 188,633 unigenes were annotated with the seven

146 databases. The results are shown in Table S2. Among the databases, $20.08 \%$ of the unigenes

147 were aligned to the $\mathrm{Nr}$ protein database with an e-value threshold of e-5 (Figure S1).

148 In total, 17,361 GO annotations were assigned to three terms: biological processes $(12,137$

149 annotations), molecular functions (1,379 annotations), and cellular component $(3,845$

150 annotations). Among the biological processes, cellular, metabolic, organic substance metabolic,

151 and primary metabolic processes were the most highly represented. The majority of the proteins

152 assigned to molecular functions were associated with binding and catalytic activity. Among the

153 cellular components, cell and intracellular proteins were the most highly represented (Figure S2).

154 KEGG pathway analysis annotated 12,456 transcripts into 320 pathways, where $24.6 \%$ of all

155 the annotated transcripts were related to metabolism, and 1922 transcripts $(15.4 \%)$ were involved

156 with signaling molecules and interaction. The cytokine-cytokine receptor interaction pathway

157 (ko04060; 804 sequences) was the most highly represented

\section{Analysis of DEGs}

159 In total, 36,221 transcripts could be detected in the combined sex transcriptomes. After strict

160 filtration, 756 DEGs were upregulated in the testis and 838 DEGs in the ovary (Figure 2). The

161 DEGs are listed in Table S3. A heatmap illustrating the hierarchical clustering of the DEGs was

162 generated to visualize the overall gene expression pattern (Figure 3). Among the 1,594 DEGs,

163 we found nine significant SEGs, as shown in Table 2.

\section{Enrichment analysis of DEGs}

165 The $1175 \mathrm{GO}$ terms were annotated as biological processes $(859 ; 73.1 \%)$, cellular component $166(109 ; 9.3 \%)$, and molecular function $(207 ; 17.6 \%)$. DEGs were assigned to 39 processes by 
167 KEGG enrichment analysis, including Cellular Processes (310 DEGs, 22.27\%), Metabolism (88

168 DEGs, 22.27\%), Genetic Information Processing (216 DEGs, 22.27\%), Environmental

169 Information Processing (352 DEGs, 22.27\%), Human Diseases (662 DEGs, 22.27\%), and

170 Organismal Systems (288 DEGs, 22.27\%). We used the same strategies (Du et al., 2017) to

171 identify genes and pathways related to gonadal development and the gametogenesis system, and

17220 significantly enriched GO terms were detected in the ovary and testis. These enriched sex-

173 related terms included male gonad development, tube development in testis, embryo

174 development, iron ion binding, and regulation of signal transduction in ovary. The 20 GO terms

175 are shown in Figure 4. In addition, 10 KEGG terms were significantly enriched in the ovary and

176 testis, i.e., the Hedgehog signaling pathway, pathways in cancer, TGF-beta signaling pathway,

177 prostate cancer in testis, metabolic pathways, Hippo signaling pathway, ovarian steroidogenesis,

178 steroid hormone biosynthesis, Wnt signaling pathway, and oocyte meiosis in ovary. The 10

179 KEGG terms are shown in Figure 5.

180 Validation of transcriptome data by real-time qRT-PCR

181 Twelve genes comprising six ovary SEGs (FGF9, FOXL2, GDF9, WNT4, CYP19A1, and

182 SAMD9), three testis SEGs (SOX9, $A M H$, and $A R$ ), and three DEGS (ARHGEF9, CKAP5, and

183 PTH2R) were selected for validation using qRT-PCR in order to confirm the reliability and

184 accuracy of the RNA-Seq method (Figure 6). The results suggested that all of the genes had 185 similar expression patterns according to both RNA-Seq and qRT-PCR, which also confirmed 186 that RNA-Seq accurately quantified the expression of genes in the ovaries and testes.

187

188 Discussion

189 In this study, we conducted the first transcriptomic gene expression analysis of adult gonads in a 
190 turtle species with TSD. In the gonads, we identify testis- and ovary-specific genes, and defined

191 the downstream gene expression cascades for both the male and female pathways (Figure 7).

192 Existence of antagonistic signals and pathways related to gonadal maintenance and 193 gametogenesis in $M$. reevesii

194 In the sexual maturity period, steroid hormones such as estrogen and androgen are necessary for

195 gonadal maintenance based on the sexually dimorphic expression of $C Y P 19 A 1$ and $A R$.

196 CYP19A1, which is a gene encoding an enzyme that catalyzes conversion from androgens to

197 estrogens (Piferrer, 2013; Matsumoto et al., 2013). CYP19A1 and other important genes such as

198 SOX9 and FOXL2, which are related to sex determination and differences, exhibited sex-

199 dimorphic expression in the early thermosensitive period (TSP) (Tang et al., 2017), and they still

200 played core roles in the adult gonads in $M$. reevesii. We also identified conserved genes with

201 known functions in sexual differentiation comprising $A M H, G D F 9$, and $W N T 4$. These genes also

202 had obvious sexually dimorphic expression patterns. The male-specific expression of $S O X 9$ 203 mRNA during fetal and adult life indicates that it has a fundamental role in testis development in 204 the turtle (Kent et al., 1996; She \& Yang, 2017). In turtles, AMH is regulated by SOX9 and this 205 pattern is consistent with $S O X 9$ upregulating $A M H$ in the mammalian testis (Bieser \& Wibbels, 206 2014). Typical ovarian-specific typical markers (FOXL2 and CYP19A1) and testicular-specific 207 marker $S O X 9$ are often expressed in a mutually exclusive manner in the gonads (Matsumoto et 208 al., 2012). FOXL2 suppresses SOX9 transcription by cooperatively binding with estrogen 209 receptors in the regulatory region of $S O X 9$ (Uhlenhaut et al., 2009). GDF9 can regulate a wide 210 range of activities in the granulosa and theca cells, including the secretion of steroid hormones

211 (Elvin et al., 2000), and it significantly suppresses the expression of $A M H$ as well as being co-

212 expressed with CYP19A1 during the sexual maturity period (Wang et al., 2017). WNT4 plays a 
213 key role in the female sexual development pathway by controlling steroidogenesis in the gonads

214 and possibly supporting oocyte development (Vainio et al., 1999), as well as repressing typical

215 male-specific steroidogenesis ( Bernard et al., 2007).

$216 D M R T 1$ is a candidate master male sex-determining gene in $T$. scripta with TSD during early

217 gonad development (Ge et al., 2017). However, in the adult turtle gonads, DMRT1 was detected

218 at low levels and it did not exhibit transcriptional sexual dimorphism between the testis and

219 ovary. Our results suggest that $D M R T 1$ does no play an important role in the adult differentiated

220 gonads. Czerwinski et al. (2016) identified six male-producing temperature-enriched genes and

221 four female-producing temperature-enriched genes with differences in expression prior to

222 DMRT1 and CYP19A1 in the early TSP. The detection of these 10 genes suggests that a gonad-

223 specific regulatory mechanism exists but it is not clear if it is causal. In our study, NOV,

224 KDM6B, RBM20, PCSK6, AVIL, and TWIST1 were detected at low levels and their expression

225 levels did not differ between the adult testis and ovary. HSPB6 was not detected. VWA2 and

$226 F D X R$ were upregulated in the ovary, but their expression levels were low in the testis, and only

227 FANK1 was strongly upregulated in the adult testis. Our results suggest that the potential gonad-

228 specific regulatory mechanism might differ between the embryo and adult.

229 The Hippo signaling pathway and Wnt signaling pathway were significantly enriched in the

230 ovary, whereas the TGF-beta signaling pathway and Hedgehog signaling pathway were

231 significantly enriched in the testis (Figure 7). The Hippo signaling pathway has critical roles in

232 controlling cell proliferation, self-renewal, differentiation, and apoptosis in most tissues and

233 organs in diverse species (Lyu et al., 2016) and it represents a molecular target for the regulation

234 of mouse ovarian functional remodeling that could be used to regulate the proliferation and

235 differentiation of ovarian function (Ye et al., 2017). Our analysis of $M$. reevesii suggested that 
236 Wnt signaling appears to function downstream of estrogen and the ovary-promoting effects of

237 the Wnt signaling pathway may be functionally conserved in mammals and reptiles. Activation

238 of the Wnt pathway antagonizes nuclear SOX9 expression (Mork et al., 2013). The TGF-beta

239 signaling pathway plays an important role in the differentiation of male germ cells in non-

240 mammalian vertebrates (Zhang et al., 2016), e.g., $A M H$ is a growth factor in the TGF-beta family

241 with a central role in testis formation. The Hedgehog signaling pathway has crucial roles in the

242 development of diverse tissue and organ systems in the embryo, and in the regulation of adult

243 tissues (Migone et al., 2017).

244 Candidate gene $F A N K 1$ might be involved with the regulation of testis spermatogenesis

245 FANK1 is exclusively expressed in the testis from the meiosis phase to the haploid phase of

246 spermatogenesis in mice, and it may have a crucial functional role in spermatogenesis as a

247 transcription factor (Dong et al., 2014). Indeed, reduced sperm numbers and increases in

248 apoptotic spermatocytes were observed in FANK1 knockdown mice. In $M$. reevesii, FANK1 was

249 strongly upregulated in the adult testis. Thus, it is possible that FANK1 play a pivotal role in

250 spermatogenesis as a transcription factor in the adult gonads. Future studies should investigate

251 the function of FANK1 during the regulation of testis maintenance.

252

253 Conclusions

254 According to our results, we propose a model where the genetic components related to steroid 255 hormones comprise networks with multiple feedback loops. The networks are antagonistic in 256 males and females, where differences in gene expression accumulate and converge to allow the 257 antagonistic regulation of steroid hormones to maintain the appropriate balance in males and 258 females. FANK1 could be involved with the regulation of testis spermatogenesis in the adult 259 turtle gonads. 


\section{Acknowledgments}

262 The authors are grateful for the comments provided by the reviewers and editors regarding the 263 manuscript.

References

Altschul SF, Gish W, Miller W, Myers EW, Lipman DJ. 1990. Basic local alignment search tool. Journal of Molecular Biology 215: 403-410 DOI 10.1016/S0022-2836(05)80360-2.

Anders S, Huber W. 2010. Differential expression analysis for sequence count data. Genome Biology 11, R106 DOI 10.1186/gb-2010-11-10-r106.

271

272

273

274

275

276

277

278

279

280

281

282

283

284

285

Benjamini Y, Hochberg Y. 1995. Controlling the false discovery rate-a practical and powerful approach to multiple testing. Journal of the Royal Statistical Society. Series B (Methodological) 57(1): 289-300.

Bernard P, Harley VR. 2007. Wnt4 action in gonadal development and sex determination. International Journal of Biochemistry \& Cell Biology 39: 31-43 DOI 10.1016/j.biocel.2006.06.007.

Bieser KL, Wibbels T. 2014. Chronology, Magnitude and Duration of Expression of Putative Sex-Determining/Differentiation Genes in a Turtle with Temperature-Dependent Sex Determination. Sexual Development 8: 364-375 DOI 10.1159/000369116.

Conesa A, Götz S, Garcíagómez JM, Terol J, Talón M, Robles M, 2005. Blast2GO: a universal tool for annotation, visualization and analysis in functional genomics research. Bioinformatics 21:3674-3676 DOI 10.1093/bioinformatics/bti610.

Czerwinski M, Natarajan A, Barske L, Looger LL, Capel B. 2016. A timecourse analysis of systemic and gonadal effects of temperature on sexual development of the red-eared slider turtle Trachemys scripta elegans. Developmental Biology 420: 166-177 DOI 
10.1016/j.ydbio.2016.09.018.

287

288

289

290

291

292

Dong WW, Huang HL, YangW, Liu J, Yu Y, Zhou SL, Wang W, Lv XC, Li ZY, Zhang MY, Zheng ZH, Yan W. 2014. Testis-specific Fankl gene in knockdown mice produces oligospermia via apoptosis. Asian journal of andrology 16(1), 124 DOI 10.4103/1008$682 X .122592$.

Du X, Wang B, Liu X, Liu X, He Y, Zhang Q, Wang X. 2017. Comparative transcriptome analysis of ovary and testis reveals potential sex-related genes and pathways in spotted Knifejaw Oplegnathus punctatus. Gene 637: 203-210 DOI 10.1016/j.gene.2017.09.055.

Elvin JA, Yan CN, Matzuk MM. 2000. Growth differentiation factor-9 stimulates progesterone synthesis in granulosa cells via a prostaglandin E2/EP2 receptor pathway. Proceedings of the National Academy of Sciences of the United States of America 97: 10288-10293 DOI $10.1073 /$ pnas. 180295197.

Ge C, Ye J, Zhang H, Zhang Y, Sun W, Sang Y, Capel B, Qian GY. 2017. Dmrt1 induces the male pathway in a turtle with temperature-dependent sex determination. Development 144: 2222-2233 DOI 10.1242/dev.152033.

Grabherr MG, Haas BJ, Yassour M, Levin JZ, Thompson DA, Amit I, Adiconis X, Fan L, Raychowdhury R, Zeng Q, Chen ZH, Mauceli E, Hacohen N, Gnirke A, Rhind N, Palma FD, Birren BW, Nusbaum C, Toh KL, Friedman N, Regev A. 2011. Full-length transcriptome assembly from RNA-Seq data without a reference genome. Nature Biotechnology 29: 644-652 DOI 10.1038/nbt.1883.

Hu P, Li G, Zhao X, Zhao F, Li L, Zhou H. 2018. Transcriptome profiling by RNA-Seq reveals differentially expressed genes related to fruit development and ripening characteristics in strawberries (Fragaria $\times$ ananassa). PeerJ, 6, e4976. DOI 
10.7717/peerj.4976.

310 Kanehisa M, Araki M, Goto S, Hattori M, Hirakawa M, Itoh M, Katayama T, Kawashima

311 S, Okuda S, Tokimatsu T. 2008. KEGG for linking genomes to life and the environment.

$312 \quad$ Nucleic Acids Research 36: 480-484 DOI 10.1093/nar/gkm882.

313 Kent J, Wheatley SC, Andrews JE, Sinclair AH, Koopman P. 1996. A male-specific role for

314 SOX9 in vertebrate sex determination. Development 122: 2813-2822.

315 Langmead B, 2010. Aligning short sequencing reads with Bowtie. Current protocols in 316 bioinformatics 32: 11-7 DOI 10.1002/0471250953.bi1107s32.

317 Li B, Dewey CN, 2011. RSEM: accurate transcript quantification from RNA-Seq data with or without a reference genome. BMC Bioinformatics 12: 323 DOI 10.1186/1471-2105-12-323.

Liu J, Jiang H, Zan J, Bao Y, Dong J, Xiong L, Nie L, 2018. Single-molecule long-read transcriptome profiling of Platysternon megacephalum mitochondrial genome with gene rearrangement and control region duplication. RNA biology 15(9): 1244-1249 DOI

Livak KJ, Schmittgen TD. 2001. Analysis of relative gene expression data using real-time quantitative PCR and the 2- $\Delta \Delta \mathrm{CT}$ method. Methods 25(4):402-408 DOI 10.1006/meth.2001.1262.

Lyu Z, Qin N, Tyasi TL, Zhu H, Liu D, Yuan S, Xu R. 2016. The Hippo/MST pathway member SAV1 plays a suppressive role in development of the prehierarchical follicles in hen ovary. PloS One 11, e0160896 DOI 10.1371/journal.pone.0160896.

Matsumoto Y, Crews D. 2012. Molecular mechanisms of temperature-dependent sex 330 determination in the context of ecological developmental biology. Molecular and Cellular Endocrinology 354: 103-110 DOI 10.1016/j.mce.2011.10.012. 
332 Matsumoto Y, Buemio A, Chu R, Vafaee M, Crews D. 2013. Epigenetic control of gonadal 333 aromatase (cyp19a1) in temperature-dependent sex determination of red-eared slider turtles.

334 PLoS One 8, e63599. DOI 10.1371/journal.pone.0063599.

335 Migone FF, Hung PH, Cowan RG, Selvaraj V, Suarez SS, Quirk SM. 2017. Overactivation

336 of hedgehog signaling in the developing Müllerian duct interferes with duct regression in 337 males and causes subfertility. Reproduction 153: 481-492 DOI 10.1530/REP-16-0562.

338 Moriya Y, Itoh M, Okuda S, Yoshizawa AC, Kanehisa M. 2007. KAAS: an automatic 339 genome annotation and pathway reconstruction server. Nucleic Acids Research 35: 182-185 $340 \quad$ DOI $10.1093 /$ nar/gkm321.

341 Mork L, Capel B. 2013. Conserved action of $\beta$-catenin during female fate determination in the 342 red-eared slider turtle. Evolution \& Development 15: 96-106 DOI 10.1111/ede.12020.

343 Piferrer F. 2013. Epigenetics of sex determination and gonadogenesis. Developmental 344 Dynamics 242(4): 360-370. DOI 10.1002/dvdy.23924.

345 She ZY, Yang WX. 2017. Sry and SoxE genes: How they participate in mammalian sex 346 determination and gonadal development? Seminars in Cell \& Developmental Biology 63: 13-22 DOI 10.1016/j.semcdb.2016.07.032.

Sun W, Cai H, Zhang G, Zhang H, Bao H, Wang L, Ye J, Qian G, Ge C. 2017. Dmrtl is required for primary male sexual differentiation in Chinese soft-shelled turtle Pelodiscus sinensis. Scientific Reports 7: 4433 DOI 10.1038/s41598-017-04938-5.

Tang WQ, Mu Y, Valenzuela N, Du WG. 2017. Effects of Incubation Temperature on the 352 Expression of Sex-Related Genes in the Chinese Pond Turtle, Mauremys reevesii. Sexual Development 11: 307-319 DOI: 10.1159/000479360.

354 Trapnell C, Williams BA, Pertea G, Mortazavi A, Kwan G, van Baren MJ, Salzberg SL, 
Wold BJ, Pachter L. 2010. Transcript assembly and quantification by RNA Seq reveals unannotated transcripts and isoform switching during cell differentiation. Nature Biotechnology 28(5):511-515 DOI 10.1038/nbt.1621.

Uhlenhaut NH, Jakob S, Anlag K, Eisenberger T, Sekido R, Kress J, Treier AC, Klugmann 2009. Somatic sex reprogramming of adult ovaries to testes by foxl2 ablation. Cell 139: 1130-1142 DOI 10.1016/j.cell.2009.11.021.

Vainio S, Heikkilä M, Kispert A, Chin N, Mcmahon AP. 1999. Female development in 363 mammals is regulated by Wnt-4 signalling. Nature 397: 405-409 DOI 10.1038/17068.

Wang Z, Qiu X, Kong D, Zhou X, Guo Z, Gao C, Shuai M, Hao WW, Jiang ZQ, Liu SC, Zhang T, Meng XS, Wang XL. 2017. Comparative RNA-seq analysis of differentially expressed genes in the testis and ovary of Takifugu rubripes. Comparative Biochemistry and Physiology Part D: Genomics and Proteomics 22: 50-57 DOI

Yatsu R, Miyagawa S, Kohno S, Parrott BB, Yamaguchi K, Ogino Y, Miyakawa H, Lowers RH, Shigenobu S, Guillette JLJ, Iguchi T. 2016. RNA-seq analysis of the gonadal transcriptome during Alligator mississippiensis, temperature-dependent sex determination

Ye H, Li X, Zheng T, Hu C, Pan Z, Huang J, Li J, Li W, Zheng Y. 2017. The Hippo Signaling Pathway Regulates Ovarian Function via the Proliferation of Ovarian Germline Stem Cells. Cellular Physiology \& Biochemistry 41: 1051-1062 DOI 10.1159/000464113. 2016. De novo assembly and characterization of the chinese three-keeled pond turtle 
378 (Mauremys reevesii) transcriptome: presence of longevity-related genes. PeerJ 4, e2062

$379 \quad$ DOI $10.7717 /$ peerj.2062.

380 Zdobnov E, Apweiler R. 2001. InterProScan - an integration platform for the signature-

381 recognition methods in InterPro. Bioinformatics 17: 847-848 DOI

$382 \quad 10.1093 /$ bioinformatics/17.9.847.

383 Zhang Y, Wang Y, Zuo Q, Li D, Zhang W, Lian C, Tang B, Xiao T, Wang M, Wang K.

384 2016. Effects of the Transforming Growth Factor Beta Signaling Pathway on the

385 Differentiation of Chicken Embryonic Stem Cells into Male Germ Cells, Cellular

386 Reprogramming 18, 401-410 DOI 10.1089/cell.2016.0019.

387

388 


\section{Figure 1}

Tissues of ovary and testis in Mauremys reevesii used in deep sequencing.

(A) Testis; (B) Ovary. Blue bar $=1000 \mu m$; Digital microscopic system VHX-5000.

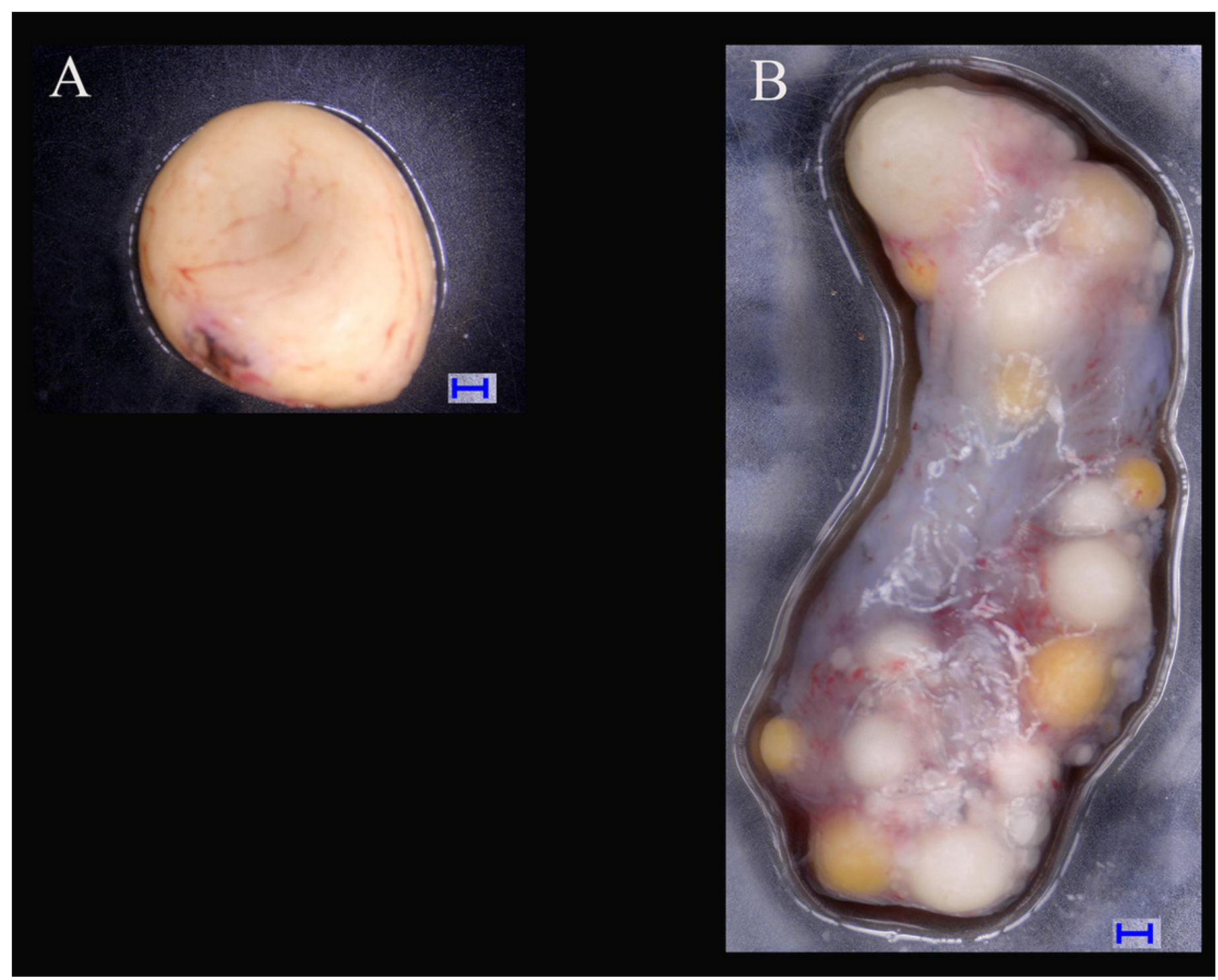


Figure 2 (on next page)

Volcanoplot where $\mathrm{X}$-axis represents the level of differential expression and theY-axis shows the significant differences in expression as negative log values.

The horizontal line is the threshold of corrected $P$ value $<0.05$. Upregulated genes in the testis are indicated by green dots, upregulated genes in the ovaries are indicated by red dots, and other genes are indicated by blue dots. 
Volcanoplot

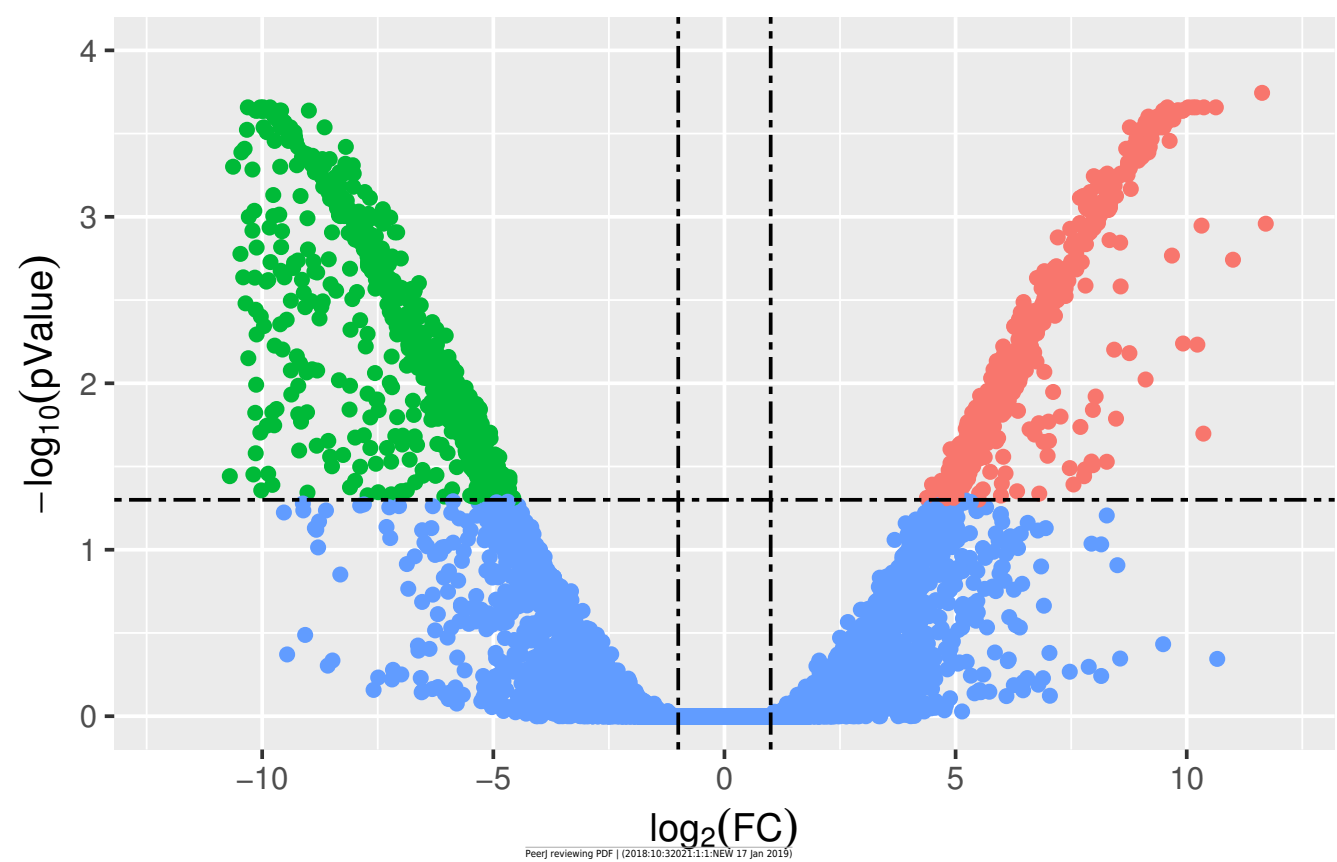

significant

- down

- no

- up 


\section{Figure 3}

Heatmap showing the DEGs in males (M1, M2, and M3) and females (F1, F2, and F3).

Blue represents weakly expressed genes and red represents highly expressed genes.

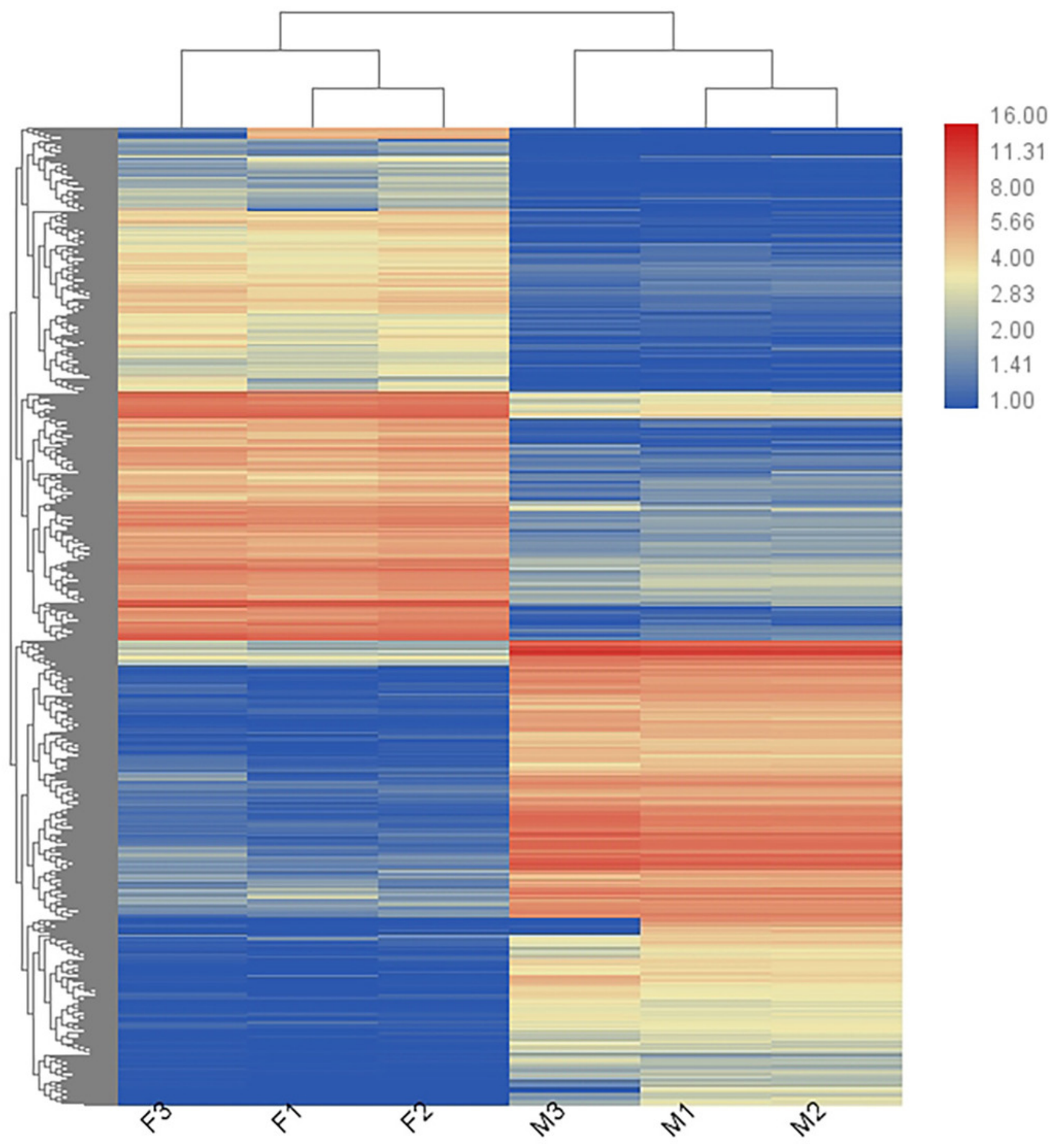


Figure 4(on next page)

Significant GO terms. 


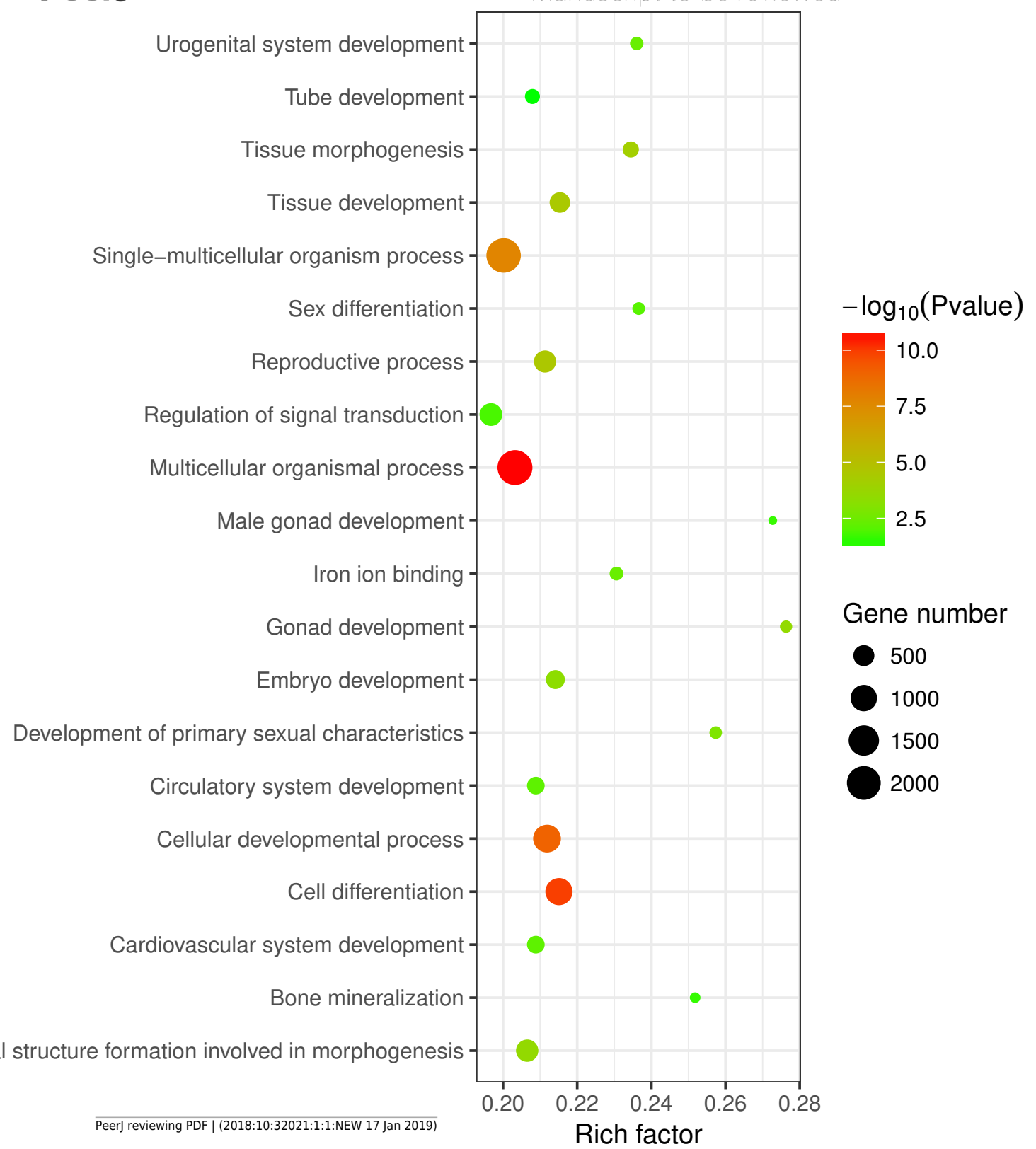


Figure $\mathbf{5}$ (on next page)

Significant KEGG terms. 


\section{PeerJ Top10 of KEGG enrichment ${ }_{\text {ancript to be reviewed }}$}

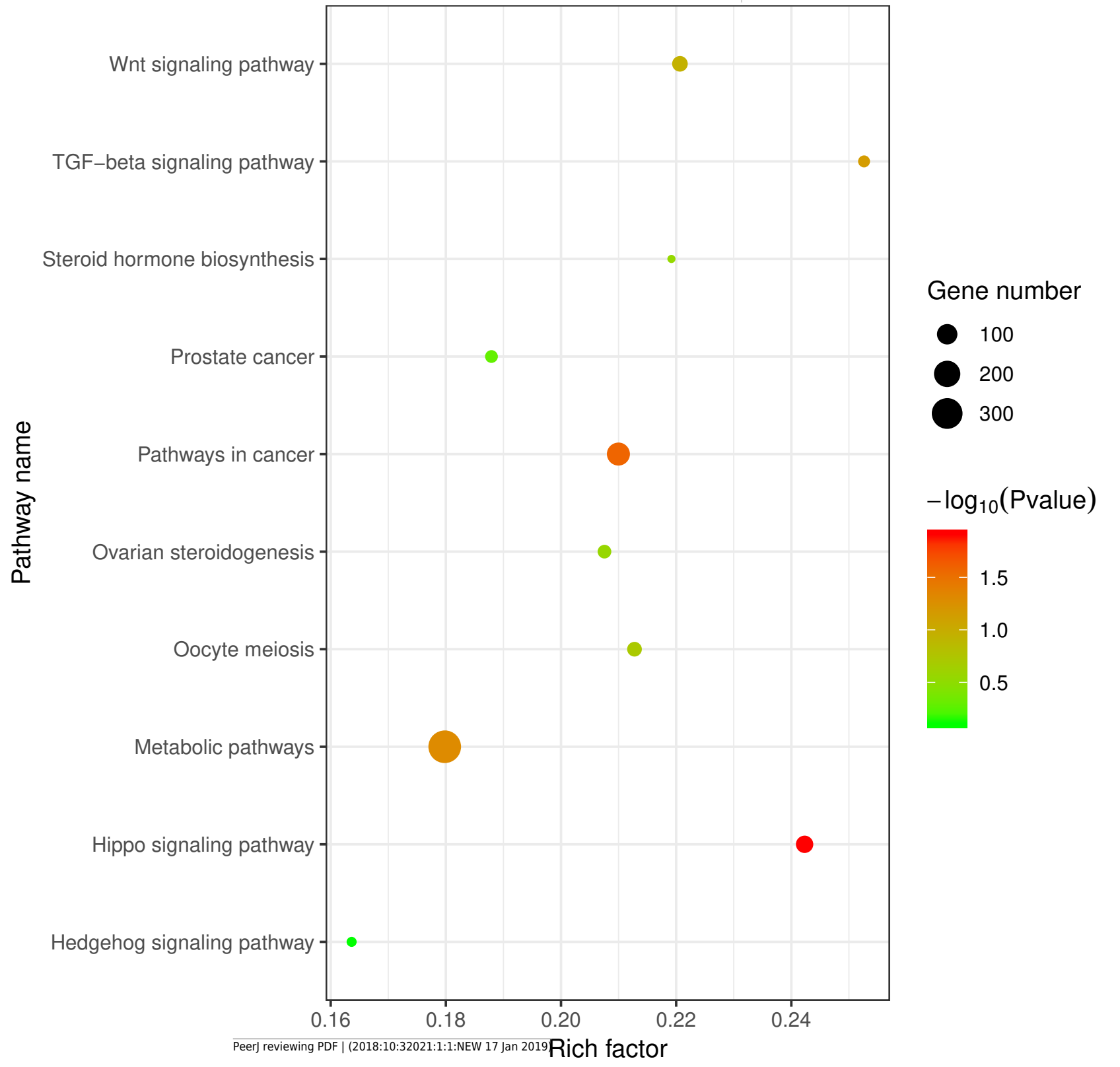


Figure 6

RNA-Seq and qRT-PCR validation results.

Expression comparisons of selected genes detected by RNA-Seq and qRT-PCR between ovary and testis. The $y$-axes show log2 (fold differences) determined by RNA-Seq and qRT-PCR. The experiments were repeated three times and provided consistent results. The mean values and error bars were obtained from three biological and three technical replicates.

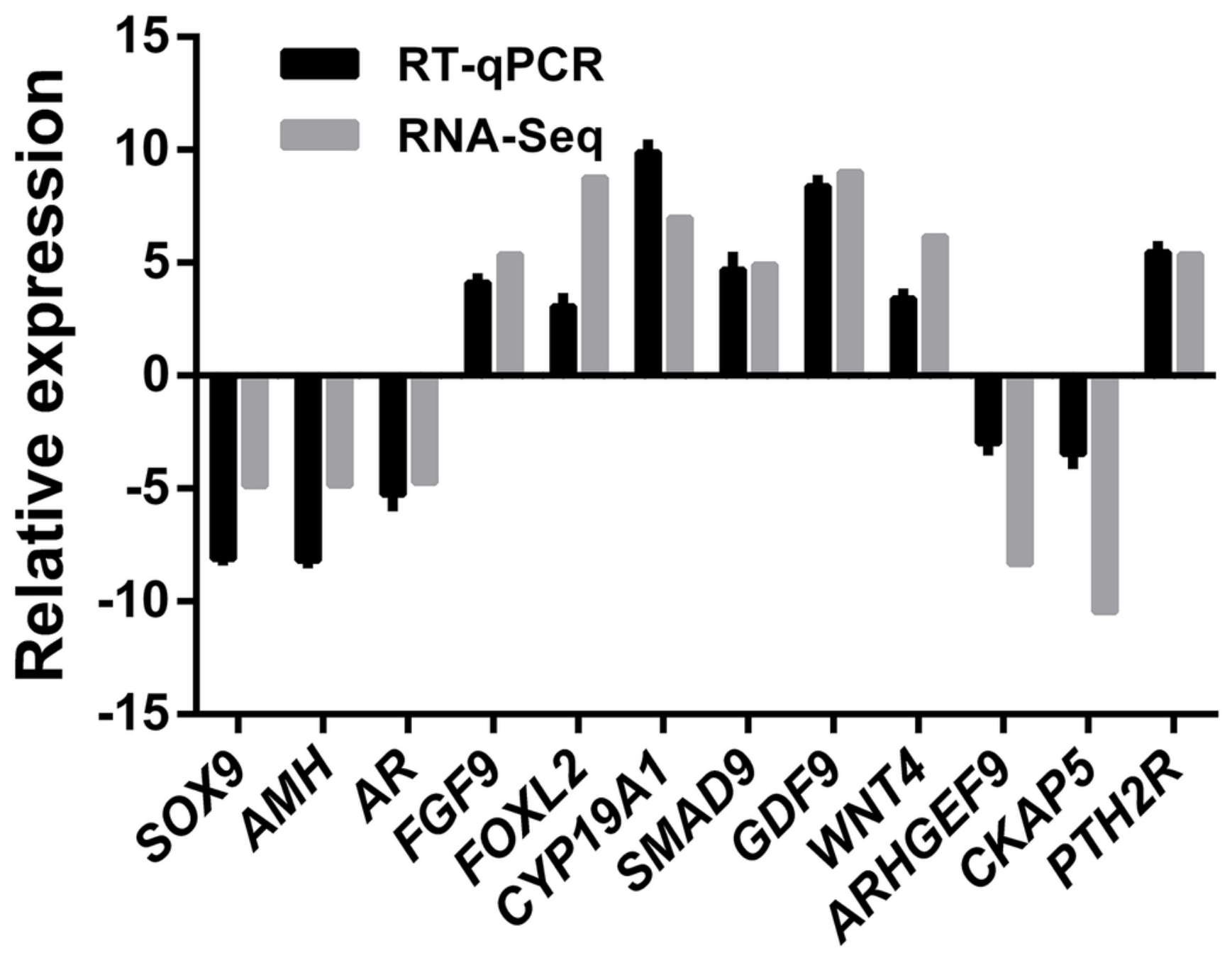


Figure 7

Putative genes and pathways involved in testis and ovary functional maintenance .

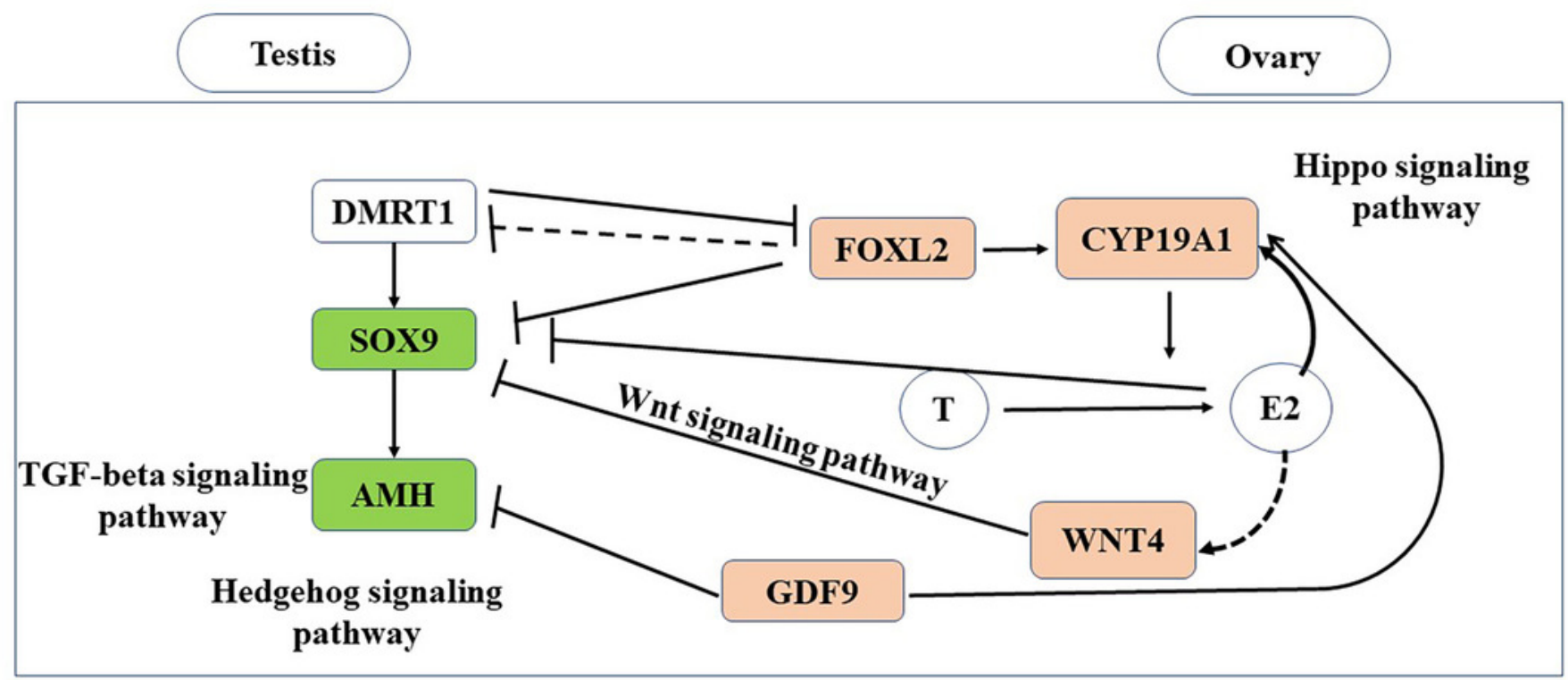




\section{Table $\mathbf{1}$ (on next page)}

Statistical analysis of the assembly quality for M.reevesii transcriptomes

(Testis: Male 1, Male 2, Male 3; Ovary: Female 1, Female 2, Female 3). 


\begin{tabular}{cccccccc}
\hline Sample & Male 1 & Male 2 & Male 3 & Female 1 & Female 2 & Female 3 \\
\hline Raw reads & $81,369,826$ & $71,156,674$ & $69,466,956$ & $50,354,874$ & $124,062,604$ & $112,374,346$ & $508,785,280$ \\
Raw bases,Mbp & 12,205 & 10,673 & 10,420 & 7,553 & 18,609 & 16,856 & 76,316 \\
Trim reads & $79,459,524$ & $69,492,528$ & $67,807,520$ & $49,205,254$ & $121,068,366$ & $109,130,658$ & $496,163,850$ \\
Trim bases,Mbp & 11,591 & 10,124 & 9,889 & 7,161 & 17,668 & 15,969 & 72,402 \\
Average length & 145.88 & 145.69 & 145.84 & 145.54 & 145.94 & 146.33 \\
Trim reads, \% & 97.65 & 97.66 & 97.61 & 97.72 & 97.59 & 97.11 \\
Trim bases, \% & 94.97 & 94.86 & 94.91 & 94.81 & 94.94 & 94.74 \\
\hline
\end{tabular}




\section{Table 2 (on next page)}

Annotation of the significant SEGs.

Testis_average: the average gene expression values of three testis biological replicates;

Ovary_average: the average gene expression values of three testis biological replicates;

log2Fold Change: the relative expression level of genes in ovary compared to that in testis ( $\log 2$ transformation); Q value: corrected $P$ value. 


\begin{tabular}{|c|c|c|c|c|c|c|}
\hline $\begin{array}{l}\text { Gene } \\
\text { name }\end{array}$ & Ensembl ID & Description & Testis_average & Ovary_average & log2Fold Change & $Q$ value \\
\hline CYP19A1 & TR102156|c1_g2_i2 & Aromatase & 3.94 & 492.55 & 6.64 & $4.58 \mathrm{E}-03$ \\
\hline FOXL2 & TR138024|c0_g1_i2 & Forkhead box protein L2 & 0.52 & 660.25 & 8.77 & $2.86 \mathrm{E}-04$ \\
\hline$A M H$ & TR109517|c0_g1_i1 & Anti-Müllerian hormone & 1526.67 & 51.75 & -4.86 & 4.44E-02 \\
\hline GDF9 & TR148262|c0_g3_i1 & $\begin{array}{c}\text { Growth/differentiation factor } \\
9\end{array}$ & 21.29 & 11568.91 & 9.02 & 4.45E-04 \\
\hline$F G F 9$ & TR108256|c0_g1_i4 & Fibroblast growth factor 9 & 9.79 & 442.85 & 5.36 & $2.25 \mathrm{E}-02$ \\
\hline SMAD9 & TR137938|c0_g1_i2 & $\begin{array}{c}\text { Mothers against } \\
\text { decapentaplegic homolog } 9\end{array}$ & 10.3 & 339.08 & 4.91 & $3.53 \mathrm{E}-02$ \\
\hline WNT4 & TR167755|c2_g1_i1 & $\begin{array}{c}\text { wingless-related MMTV } \\
\text { integration site } 4\end{array}$ & 105.49 & 7614.18 & 6.16 & 7.14E-03 \\
\hline SOX9 & TR144387|c0_g2_i1 & Transcription factor SOX-9 & 1637.35 & 54.36 & -4.89 & $4.05 \mathrm{E}-02$ \\
\hline$A R$ & TR166731|c0_g1_i3 & Androgen receptor & 32.95 & 0.26 & -4.75 & 4.37E-02 \\
\hline
\end{tabular}

